Supplement of Biogeosciences, 16, 3835-3852, 2019

https://doi.org/10.5194/bg-16-3835-2019-supplement

(C) Author(s) 2019. This work is distributed under

the Creative Commons Attribution 4.0 License.

(c) (1)

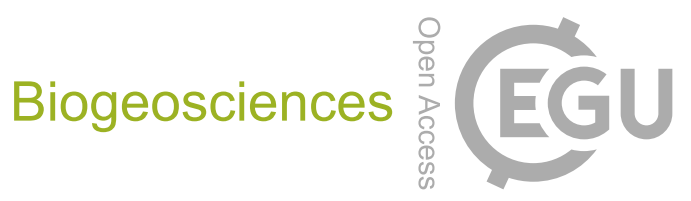

Supplement of

\title{
Rapid environmental responses to climate-induced hydrographic changes in the Baltic Sea entrance
}

Laurie M. Charrieau et al.

Correspondence to: Helena L. Filipsson (helena.filipsson@geol.lu.se)

The copyright of individual parts of the supplement might differ from the CC BY 4.0 License. 

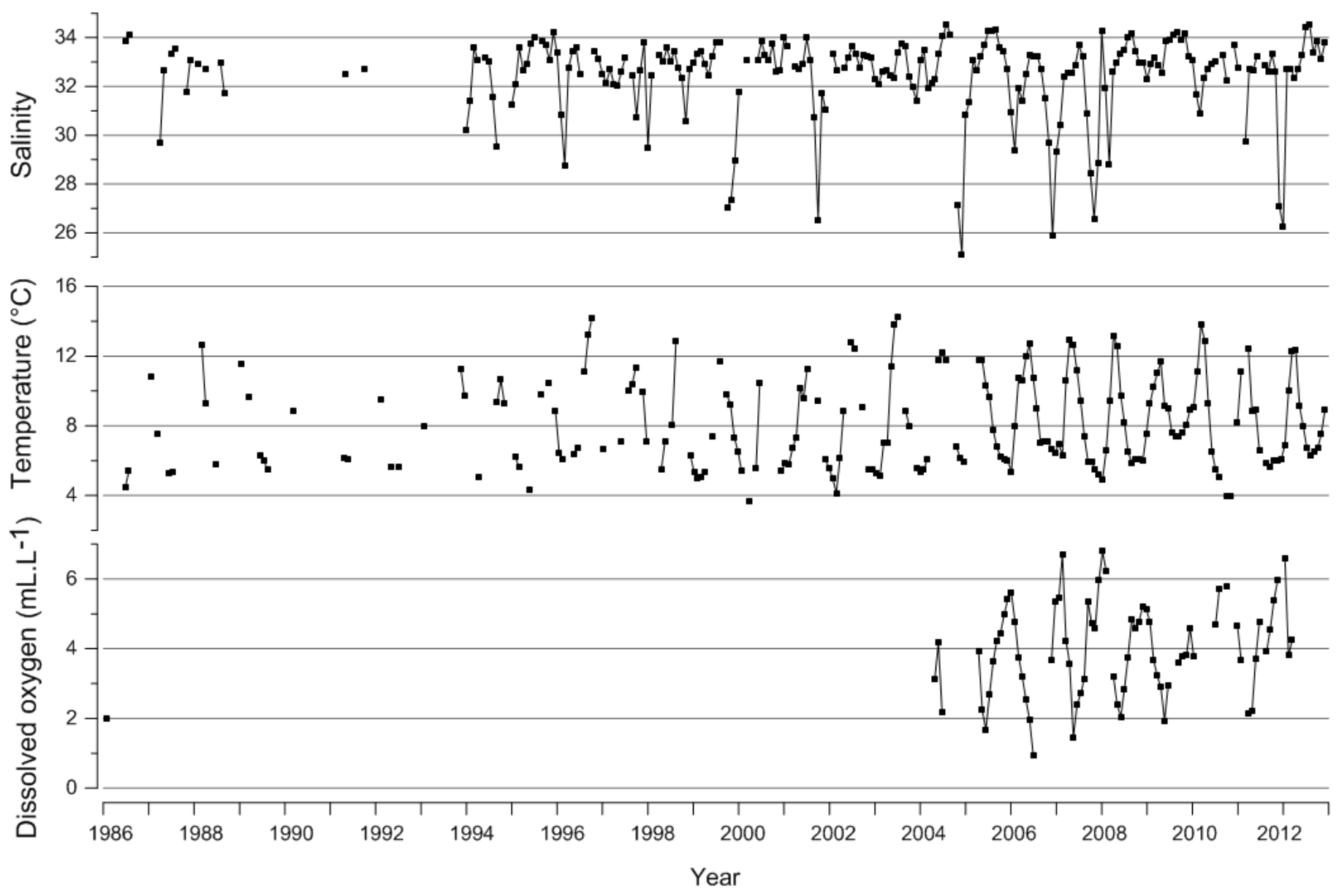

Supplement Fig. S1. Time series of salinity, temperature and dissolved oxygen concentration at the bottom water (40 m) of the Öresund between 1986 and 2013. The data were measured by the SMHI (Swedish Meteorological and Hydrological Institute) at the station W LANDSKRONA. 
Supplement Table S1. Total faunas, normalized to $50 \mathrm{~cm}^{3}$

\begin{tabular}{|c|c|c|c|c|c|c|c|c|c|c|c|c|c|c|c|c|c|c|c|c|c|c|}
\hline \multirow{4}{*}{$\begin{array}{l}\text { Station name } \\
\text { FOR zones } \\
\text { Centimeter } \\
\text { Species } \\
\end{array}$} & \multicolumn{22}{|c|}{ DV } \\
\hline & \multicolumn{2}{|c|}{ FOR-C } & \multicolumn{3}{|c|}{ FOR-B2 } & \multicolumn{7}{|c|}{ FOR-B1 } & \multicolumn{6}{|c|}{ FOR-A2 } & \multicolumn{4}{|c|}{ FOR-A1 } \\
\hline & 1 & 2 & 4 & 5 & 6 & 8 & 10 & 12 & 14 & 15 & 16 & 17 & 18 & 20 & 22 & 24 & 26 & 28 & 30 & 32 & 34 & 36 \\
\hline & & & & & & & & & & & & & & & & & & & & & & \\
\hline Biloculinella inflata & 6 & 13 & 0 & 0 & 0 & 0 & 0 & 0 & 0 & 0 & 0 & 0 & 0 & 0 & 0 & 0 & 0 & 0 & 0 & 0 & 0 & 0 \\
\hline Cornuspira involvens & 0 & 6 & 9 & 0 & 0 & 0 & 0 & 0 & 0 & 0 & 0 & 0 & 0 & 0 & 0 & 0 & 0 & 7 & 0 & 0 & 0 & 0 \\
\hline Pyrgo williamsoni & 1 & 1 & 0 & 16 & 0 & 0 & 0 & 0 & 0 & 0 & 0 & 0 & 0 & 0 & 0 & 0 & 0 & 28 & 0 & 0 & 0 & 0 \\
\hline Quinqueloculina seminula & 0 & 1 & 0 & 0 & 35 & 0 & 14 & 0 & 0 & 0 & 30 & 0 & 0 & 0 & 0 & 0 & 0 & 0 & 0 & 0 & 0 & 0 \\
\hline Quinqueloculina stalkeri & 6 & 0 & 9 & 0 & 18 & 0 & 0 & 0 & 0 & 0 & 0 & 0 & 0 & 0 & 0 & 0 & 7 & 14 & 0 & 0 & 0 & 0 \\
\hline Porcelaneous varia & 13 & 31 & 111 & 32 & 0 & 0 & 0 & 0 & 34 & 8 & 46 & 0 & 0 & 8 & 0 & 0 & 0 & 0 & 7 & 9 & 0 & 0 \\
\hline Organic linings & 0 & 0 & 146 & 159 & 158 & 60 & 345 & 132 & 171 & 238 & 304 & 332 & 686 & 575 & 599 & 807 & 444 & 260 & 608 & 316 & 649 & 159 \\
\hline Ammonia beccarii & 0 & 0 & 292 & 191 & 308 & 105 & 159 & 822 & 1495 & 2167 & 1033 & 498 & 123 & 121 & 15 & 16 & 14 & 49 & 57 & 103 & 56 & 25 \\
\hline Ammonia falsobeccarii & 57 & 77 & 69 & 80 & 35 & 37 & 111 & 350 & 854 & 986 & 516 & 231 & 85 & 23 & 15 & 0 & 0 & 0 & 0 & 0 & 0 & 0 \\
\hline Ammonia spp. & 0 & 0 & 0 & 0 & 0 & 142 & 0 & 0 & 0 & 0 & 0 & 0 & 0 & 0 & 0 & 0 & 0 & 0 & 0 & 0 & 0 & 0 \\
\hline Bolivina pseudoplicata & 0 & 0 & 9 & 0 & 0 & 0 & 0 & 0 & 68 & 0 & 0 & 0 & 0 & 0 & 0 & 0 & 0 & 0 & 0 & 0 & 0 & 2 \\
\hline Bolivina pseudopunctata & 19 & 0 & 0 & 0 & 0 & 0 & 0 & 0 & 0 & 0 & 0 & 0 & 0 & 0 & 0 & 0 & 0 & 0 & 0 & 0 & 0 & 0 \\
\hline Bolivina spp. & 0 & 0 & 9 & 0 & 0 & 0 & 0 & 19 & 0 & 0 & 0 & 0 & 0 & 0 & 0 & 0 & 0 & 0 & 0 & 0 & 0 & 0 \\
\hline Bulimina marginata & 132 & 107 & 506 & 414 & 282 & 187 & 166 & 661 & 1128 & 1224 & 501 & 534 & 116 & 68 & 29 & 16 & 57 & 7 & 0 & 0 & 8 & 6 \\
\hline Buliminella elegantissima & 0 & 6 & 206 & 143 & 176 & 60 & 83 & 57 & 103 & 170 & 61 & 29 & 8 & 8 & 7 & 8 & 0 & 70 & 7 & 9 & 0 & 0 \\
\hline Cassidulina laevigata & 44 & 101 & 300 & 112 & 35 & 22 & 0 & 340 & 376 & 510 & 228 & 116 & 15 & 8 & 7 & 0 & 0 & 0 & 0 & 0 & 0 & 2 \\
\hline Cassidulina reniforme & 0 & 13 & 17 & 32 & 0 & 15 & 14 & 19 & 68 & 0 & 15 & 14 & 0 & 0 & 0 & 0 & 0 & 0 & 0 & 0 & 0 & 0 \\
\hline Cibicides lobatulus & 63 & 57 & 352 & 287 & 211 & 22 & 41 & 359 & 410 & 238 & 273 & 130 & 8 & 8 & 0 & 16 & 0 & 7 & 14 & 43 & 8 & 8 \\
\hline Elphidium albiumbilicatum & 25 & 63 & 489 & 143 & 528 & 225 & 180 & 454 & 410 & 238 & 213 & 217 & 77 & 53 & 15 & 31 & 29 & 127 & 78 & 77 & 0 & 14 \\
\hline Elphidium clavatum & 201 & 289 & 986 & 1833 & 2077 & 809 & 567 & 1436 & 2631 & 3331 & 1018 & 1430 & 154 & 136 & 51 & 39 & 100 & 183 & 155 & 111 & 72 & 45 \\
\hline Elphidium magellanicum & 63 & 94 & 292 & 223 & 528 & 135 & 180 & 529 & 547 & 408 & 349 & 130 & 62 & 45 & 0 & 0 & 43 & 141 & 92 & 60 & 8 & 8 \\
\hline Elphidium williamsoni & 19 & 19 & 86 & 32 & 18 & 22 & 14 & 113 & 68 & 136 & 61 & 14 & 0 & 0 & 0 & 0 & 7 & 28 & 21 & 51 & 16 & 6 \\
\hline Elphidium spp. & 69 & 126 & 86 & 0 & 53 & 0 & 28 & 0 & 0 & 0 & 0 & 0 & 0 & 15 & 0 & 0 & 21 & 7 & 14 & 17 & 8 & 2 \\
\hline Epistominella vitrea & 19 & 13 & 309 & 367 & 299 & 120 & 166 & 227 & 103 & 204 & 30 & 43 & 23 & 0 & 7 & 0 & 7 & 0 & 0 & 0 & 0 & 0 \\
\hline Fissurina spp. & 0 & 0 & 0 & 0 & 0 & 0 & 0 & 0 & 34 & 0 & 15 & 0 & 0 & 0 & 0 & 0 & 0 & 0 & 0 & 0 & 0 & 0 \\
\hline Parafissurina spp. & 0 & 0 & 43 & 16 & 35 & 22 & 0 & 38 & 34 & 68 & 15 & 14 & 8 & 0 & 0 & 0 & 0 & 7 & 0 & 0 & 0 & 0 \\
\hline Fursenkoïna spp. & 0 & 0 & 0 & 0 & 0 & 0 & 0 & 0 & 0 & 0 & 0 & 0 & 0 & 0 & 0 & 0 & 0 & 7 & 0 & 0 & 0 & 0 \\
\hline Gavelinopsis praegeri & 0 & 6 & 0 & 0 & 0 & 0 & 0 & 0 & 0 & 0 & 15 & 0 & 0 & 0 & 0 & 0 & 0 & 0 & 0 & 0 & 0 & 0 \\
\hline Giroidina sp. & 0 & 0 & 0 & 0 & 0 & 0 & 0 & 0 & 34 & 0 & 0 & 0 & 0 & 0 & 0 & 0 & 0 & 0 & 0 & 0 & 0 & 0 \\
\hline Haynesina depressula & 25 & 25 & 51 & 0 & 0 & 0 & 0 & 0 & 0 & 0 & 0 & 0 & 0 & 0 & 0 & 0 & 0 & 0 & 0 & 17 & 0 & 0 \\
\hline Hyalinea balthica & 0 & 19 & 9 & 0 & 0 & 7 & 0 & 0 & 34 & 0 & 0 & 0 & 0 & 0 & 0 & 0 & 0 & 0 & 0 & 0 & 0 & 0 \\
\hline Lagena laevis & 0 & 0 & 0 & 0 & 0 & 7 & 0 & 0 & 0 & 0 & 15 & 0 & 0 & 0 & 7 & 0 & 7 & 14 & 0 & 0 & 0 & 0 \\
\hline
\end{tabular}


Supplement Table S1. Total faunas, normalized to $50 \mathrm{~cm}^{3}$

Lagena semistriata

Lagena substriata

Lagena sulcata

Lenticulina sp.

Loxostomum sp.

Nonionella sp. T1

Nonionella iridea

Nonionellina labradorica

Nonionoides turgida

Nonionella spp.

Nonionellina spp.

Oolina melo

Polymorphina spp.

Procerolagena clavata

Procerolagena grassilima

Procerolagena mollis

Robertina arctica

Rosalina spp.

Stainforthia fusiformis

Stainforthia loeblichi

Hyalin indet (round)

Hyalin indet (twisted)

Hyalin varia

Adercotryma glomerata

Ammodiscus sp.

Ammoscalaria pseudospiralis

Ammotium cassis

Cribrostomoides crassimargo

Cribrostomoides subglobosum

Cribrostomoides spp.

Recurvoides spp.

Eggerelloides medius/scabrus

Haplophragmoides bradyi

Lagenammina difflugiformis

Leptohalysis scotti

Miliammina fusca

\begin{tabular}{|c|c|c|c|c|c|c|c|c|c|c|c|c|c|c|c|c|c|c|c|c|c|}
\hline 0 & 0 & 0 & 0 & 18 & 0 & 0 & 0 & 0 & 0 & 0 & 0 & 0 & 8 & 7 & 0 & 0 & 0 & 0 & 0 & 0 & 0 \\
\hline 0 & 13 & 9 & 32 & 53 & 22 & 14 & 0 & 34 & 34 & 0 & 0 & 15 & 15 & 0 & 8 & 14 & 14 & 0 & 9 & 0 & 0 \\
\hline 0 & 0 & 9 & 0 & 18 & 0 & 0 & 0 & 103 & 34 & 0 & 0 & 8 & 0 & 0 & 0 & 0 & 0 & 0 & 9 & 0 & 0 \\
\hline 0 & 0 & 0 & 48 & 0 & 7 & 0 & 0 & 0 & 34 & 0 & 0 & 0 & 0 & 7 & 0 & 0 & 0 & 0 & 9 & 8 & 0 \\
\hline 0 & 0 & 9 & 0 & 0 & 0 & 0 & 57 & 0 & 0 & 0 & 0 & 0 & 0 & 0 & 0 & 0 & 7 & 0 & 0 & 0 & 2 \\
\hline 308 & 176 & 94 & 0 & 18 & 0 & 0 & 0 & 0 & 0 & 0 & 0 & 0 & 0 & 0 & 0 & 0 & 0 & 0 & 0 & 0 & 0 \\
\hline 0 & 0 & 0 & 16 & 18 & 22 & 0 & 38 & 0 & 0 & 0 & 0 & 0 & 0 & 0 & 0 & 0 & 21 & 0 & 0 & 0 & 0 \\
\hline 113 & 75 & 249 & 143 & 141 & 135 & 97 & 340 & 513 & 382 & 243 & 188 & 54 & 23 & 22 & 16 & 29 & 56 & 106 & 103 & 40 & 12 \\
\hline 138 & 189 & 103 & 64 & 106 & 0 & 0 & 38 & 34 & 34 & 15 & 0 & 0 & 0 & 0 & 0 & 0 & 7 & 0 & 0 & 0 & 2 \\
\hline 0 & 0 & 0 & 16 & 35 & 0 & 0 & 19 & 0 & 0 & 0 & 0 & 8 & 0 & 0 & 0 & 0 & 7 & 0 & 0 & 0 & 0 \\
\hline 0 & 0 & 17 & 0 & 0 & 0 & 0 & 19 & 34 & 0 & 0 & 0 & 0 & 0 & 0 & 0 & 0 & 0 & 0 & 0 & 0 & 2 \\
\hline 6 & 0 & 0 & 0 & 0 & 0 & 0 & 19 & 0 & 68 & 0 & 0 & 0 & 0 & 0 & 0 & 0 & 0 & 0 & 0 & 0 & 0 \\
\hline 0 & 0 & 9 & 16 & 0 & 0 & 0 & 38 & 0 & 0 & 15 & 0 & 0 & 15 & 0 & 0 & 7 & 0 & 7 & 9 & 0 & 0 \\
\hline 0 & 6 & 0 & 0 & 0 & 0 & 0 & 0 & 0 & 0 & 0 & 0 & 0 & 0 & 0 & 0 & 0 & 0 & 0 & 0 & 0 & 0 \\
\hline 0 & 0 & 43 & 0 & 18 & 15 & 0 & 0 & 0 & 0 & 30 & 14 & 8 & 23 & 7 & 0 & 0 & 0 & 0 & 9 & 0 & 0 \\
\hline 0 & 0 & 17 & 0 & 0 & 0 & 0 & 0 & 0 & 0 & 0 & 0 & 8 & 8 & 0 & 0 & 0 & 0 & 7 & 0 & 0 & 0 \\
\hline 0 & 6 & 0 & 0 & 0 & 0 & 0 & 0 & 0 & 0 & 0 & 0 & 0 & 0 & 0 & 0 & 0 & 0 & 0 & 0 & 0 & 0 \\
\hline 0 & 0 & 0 & 32 & 0 & 0 & 0 & 0 & 0 & 102 & 0 & 0 & 0 & 0 & 0 & 0 & 0 & 0 & 0 & 0 & 0 & 0 \\
\hline 126 & 119 & 746 & 669 & 827 & 277 & 373 & 340 & 547 & 306 & 258 & 838 & 1025 & 2029 & 402 & 541 & 1096 & 2112 & 1144 & 427 & 304 & 161 \\
\hline 0 & 0 & 17 & 16 & 0 & 0 & 0 & 0 & 0 & 0 & 0 & 0 & 8 & 0 & 0 & 8 & 7 & 0 & 7 & 0 & 16 & 0 \\
\hline 0 & 0 & 9 & 0 & 0 & 0 & 0 & 0 & 68 & 68 & 15 & 14 & 15 & 0 & 0 & 0 & 7 & 14 & 14 & 0 & 0 & 2 \\
\hline 0 & 0 & 17 & 0 & 18 & 0 & 0 & 38 & 0 & 34 & 30 & 0 & 8 & 0 & 0 & 0 & 0 & 0 & 0 & 0 & 0 & 0 \\
\hline 6 & 0 & 0 & 0 & 0 & 0 & 0 & 0 & 0 & 34 & 0 & 0 & 0 & 0 & 0 & 0 & 0 & 0 & 0 & 0 & 0 & 2 \\
\hline 13 & 44 & 206 & 127 & 35 & 0 & 14 & 0 & 0 & 0 & 0 & 0 & 15 & 0 & 0 & 0 & 0 & 0 & 0 & 0 & 0 & 0 \\
\hline 0 & 0 & 9 & 32 & 18 & 0 & 0 & 0 & 0 & 0 & 0 & 0 & 0 & 0 & 0 & 0 & 0 & 0 & 0 & 0 & 0 & 2 \\
\hline 6 & 0 & 51 & 8 & 53 & 22 & 41 & 189 & 589 & 484 & 319 & 65 & 8 & 8 & 15 & 0 & 0 & 0 & 14 & 9 & 0 & 0 \\
\hline 1 & 0 & 0 & 80 & 18 & 0 & 0 & 0 & 0 & 0 & 0 & 0 & 0 & 0 & 0 & 0 & 0 & 0 & 0 & 0 & 0 & 0 \\
\hline 0 & 0 & 17 & 16 & 106 & 30 & 28 & 0 & 0 & 0 & 0 & 0 & 0 & 0 & 0 & 0 & 0 & 14 & 0 & 9 & 0 & 2 \\
\hline 0 & 2 & 0 & 0 & 0 & 0 & 0 & 19 & 0 & 0 & 0 & 0 & 0 & 0 & 0 & 0 & 7 & 0 & 0 & 0 & 0 & 0 \\
\hline 0 & 0 & 206 & 207 & 317 & 45 & 69 & 19 & 103 & 170 & 46 & 116 & 62 & 38 & 44 & 16 & 21 & 28 & 14 & 26 & 16 & 2 \\
\hline 57 & 44 & 0 & 0 & 53 & 0 & 28 & 0 & 0 & 0 & 0 & 0 & 0 & 0 & 0 & 0 & 0 & 0 & 0 & 0 & 0 & 0 \\
\hline 189 & 170 & 1055 & 1115 & 986 & 847 & 1133 & 4327 & 7756 & 9279 & 5696 & 3769 & 1125 & 712 & 920 & 470 & 516 & 514 & 1349 & 1325 & 793 & 223 \\
\hline 6 & 0 & 0 & 0 & 0 & 0 & 0 & 0 & 0 & 0 & 0 & 0 & 0 & 0 & 0 & 0 & 0 & 0 & 0 & 0 & 0 & 0 \\
\hline 25 & 6 & 26 & 0 & 70 & 0 & 0 & 0 & 26 & 0 & 76 & 0 & 0 & 0 & 7 & 8 & 7 & 14 & 0 & 17 & 0 & 8 \\
\hline 63 & 25 & 0 & 0 & 0 & 0 & 0 & 0 & 0 & 0 & 0 & 0 & 0 & 0 & 0 & 0 & 0 & 0 & 0 & 0 & 0 & 0 \\
\hline 0 & 0 & 26 & 32 & 0 & 7 & 0 & 19 & 0 & 102 & 0 & 0 & 0 & 23 & 0 & 0 & 7 & 21 & 7 & 9 & 0 & 2 \\
\hline
\end{tabular}


Supplement Table S1. Total faunas, normalized to $50 \mathrm{~cm}^{3}$

Paratrochammina haynesi

Psammosphaera bowmann

Reophax subfusiformis

Reophax spp.

Spiroplectammina biformis

Textularia earlandi

Textularia kattegatensis

Textularia spp.

Rotaliammina adaperta

Trochammina spp.

Agglutinated varia

TOTAL

\begin{tabular}{|rr|rrr|rrrrrrr|rrrrrrrrrrrr}
0 & 0 & 0 & 0 & 0 & 0 & 0 & 0 & 0 & 102 & 0 & 14 & 0 & 0 & 0 & 0 & 0 & 0 & 0 & 0 & 0 & 0 \\
6 & 0 & 0 & 0 & 18 & 0 & 14 & 0 & 0 & 0 & 0 & 0 & 0 & 8 & 0 & 0 & 0 & 0 & 0 & 0 & 0 & 0 \\
285 & 181 & 583 & 430 & 722 & 127 & 207 & 557 & 1102 & 1198 & 440 & 173 & 139 & 106 & 153 & 39 & 29 & 56 & 92 & 60 & 32 & 27 \\
0 & 0 & 0 & 0 & 0 & 0 & 14 & 0 & 0 & 0 & 0 & 0 & 0 & 0 & 0 & 0 & 0 & 0 & 0 & 0 & 0 & 0 \\
19 & 50 & 343 & 207 & 282 & 30 & 138 & 0 & 0 & 0 & 0 & 0 & 62 & 83 & 22 & 47 & 43 & 42 & 35 & 0 & 0 & 20 \\
57 & 0 & 60 & 0 & 88 & 0 & 0 & 0 & 0 & 0 & 0 & 0 & 0 & 8 & 0 & 0 & 0 & 7 & 0 & 0 & 0 & 0 \\
0 & 6 & 0 & 0 & 0 & 0 & 0 & 0 & 0 & 0 & 0 & 0 & 0 & 0 & 0 & 0 & 0 & 0 & 0 & 0 & 0 & 0 \\
0 & 0 & 26 & 0 & 0 & 7 & 0 & 0 & 0 & 0 & 0 & 0 & 0 & 0 & 0 & 0 & 0 & 0 & 0 & 0 & 0 & 0 \\
0 & 0 & 0 & 32 & 53 & 22 & 41 & 0 & 34 & 68 & 46 & 14 & 8 & 23 & 15 & 0 & 29 & 21 & 21 & 9 & 8 & 12 \\
0 & 0 & 0 & 0 & 53 & 0 & 28 & 19 & 0 & 102 & 46 & 29 & 8 & 0 & 0 & 24 & 0 & 21 & 0 & 9 & 0 & 0 \\
6 & 19 & 137 & 0 & 0 & 0 & 0 & 76 & 0 & 0 & 0 & 0 & 100 & 114 & 139 & 78 & 136 & 77 & 92 & 0 & 104 & 31 \\
2192 & 2198 & 8472 & 7418 & 8933 & 3620 & 4304 & 11725 & 19544 & 22561 & 12015 & 8968 & 4045 & 4308 & 2511 & 2187 & 2694 & 4013 & 3963 & 2854 & 2147 & 788
\end{tabular}

